\title{
Model-Based Optical Resolution
}

\author{
Arnold J. den Dekker
}

\begin{abstract}
This paper considers the two-point resolution of imaging systems from the viewpoint of model fitting theory. Earlier results have been extended to include (partially) coherent sources of unknown intensity. Furthermore, it is shown how the probability of resolution can be computed. This probability can be used to compare the performance of different imaging systems.
\end{abstract}

Index Terms-Bifurcation, image resolution, image restoration, modeling, nonlinear estimation, parameter estimation.

\section{INTRODUCTION}

$\mathbf{T}$ WO-POINT resolution is defined as the ability of an imaging system to separate two point sources of equal intensity. It is a widely used measure in the quality assessment of imaging systems. Following the approach of [1], two kinds of images can be distinguished: calculated and detected images. Calculated images are by their very nature noise-free and exactly describable by a known mathematical two-component model. The generally accepted classical resolution criteria, like Rayleigh's [2], concern such calculated images and provide resolution limits that are a measure of the width of the point spread function (PSF) of the diffraction limited imaging system. However, if calculated images would exist, one could numerically fit the two-component model to the observations with respect to the component amplitudes and locations, the resulting fit would be perfect and the resolution would be infinitely high and not restricted by the width of the PSF. Obviously, to obtain practical information about the resolving power of imaging systems, one should consider detected images, since these are the only kind of images encountered in practice. For detected images, fitting a two-component model will never result in a perfect fit and resolution will be limited. This is caused by both systematic errors (differences between the model fitted and the one underlying the observations) and nonsystematic errors (noise) [3]. Many modern resolution criteria are based on asymptotic statistical parameter estimation theory [4]-[7]. Under the assumptions that i) the model is properly specified, i.e., there are no systematic errors, ii) the probability function of the nonsystematic errors is known and iii) a very large number of observations is available, this theory provides expressions for the so-called Cramér-Rao Lower Bounds (CRLB's) on the variance of estimators of object parameters like the locations and the intensities of point sources. These CRLB's are a measure of the attainable precision and, therefore, of resolution. The CRLB's are asymptotically attained by a maximum likeli-

Manuscript received June 3, 1996.

The author is with the Department of Applied Physics, Delft University of Technology, 2628 CJ Delft, The Netherlands (e-mail: dekker@tn.tudelft.nl). Publisher Item Identifier S 0018-9456(97)06470-X. hood estimator and decrease with increasing signal-to-noise ratio (SNR). However, the assumptions mentioned above are often not realistic in practice. In this paper an alternative parameter estimation based definition of two-point resolution is presented, which does not make these assumptions.

Earlier results on parametric model-based optical resolution [8]-[9] have been extended to include (partially) coherent sources of unknown intensity. A further generalization is that the two-component model underlying the observations and that chosen by the experimenter need not be the same. Furthermore, it is shown how the probability of resolution as a function of the SNR can be computed. Special attention is paid to imaging systems with a Gaussian PSF, since in practice many PSF's can be well approximated by a Gaussian one [10].

\section{THE PARAMETRIC MODEL AND THE CRITERION OF GOODNESS OF FIT}

Suppose that a set of observations $\left(w_{1}, \ldots, w_{N}\right)$ on a one-dimensional (1-D) composite intensity distribution in the image of two point sources is available and that the following two-point image model [11]

$$
\begin{aligned}
g_{n}\left(a, \ell, b_{1}, b_{2}\right)= & a\left[\ell^{2} f^{2}\left(x_{n}-b_{1}\right)+(1-\ell)^{2} f^{2}\left(x_{n}-b_{2}\right)\right. \\
& \left.+2 \gamma \ell(1-\ell) f\left(x_{n}-b_{1}\right) f\left(x_{n}-b_{2}\right)\right]
\end{aligned}
$$

is fitted to the observations with respect to the parameters $a, \ell$, $b_{1}$, and $b_{2}$. In (1), $f(x)$ is the amplitude PSF, $a \ell^{2}$ and $a(1-\ell)^{2}$ are the peak intensities of the individual point images, $b_{1}$ and $b_{2}$ are the locations, and $\gamma$ is the real part of the complex degree of coherence, with $|\gamma| \leq 1$ [11]. The parameter $\ell$ determines the peak intensity ratio. The variables $x_{n}$ are the independent spatial variables (measurement points), which are assumed to be known. The model is fitted in the sense of least squares (LS). This means that the LS criterion

$$
J_{2}\left(a, \ell, b_{1}, b_{2}\right)=\sum_{n} d_{n}^{2}\left(a, \ell, b_{1}, b_{2}\right)
$$

is minimized with respect to $\left(a, \ell, b_{1}, b_{2}\right)$, where $d_{n}\left(a, \ell, b_{1}, b_{2}\right)=w_{n}-g_{n}\left(a, \ell, b_{1}, b_{2}\right)$. Furthermore, in (2) the subscript 2 refers to the fact that a $t w o$-component model such as the model (1) is fitted. To simplify the procedure, the minimization with respect to $\left(a, \ell, b_{1}, b_{2}\right)$ is replaced by minimization with respect to $\left(a, b_{1}, b_{2}\right)$ for all relevant values of $\ell$. These are usually all values on a subinterval of the interval $(0,1)$ [12]-[13]. The best fit is taken as a solution. If a priori knowledge about the ratio of the peak intensities $a \ell^{2}$ and $a(1-\ell)^{2}$ is available, this can be used directly by determining the value of $\ell$. By definition, two-point resolution concerns two point sources of equal intensity. Accordingly, considering two-point resolution, 
one should impose a constraint on $\ell$, since a solution consisting of a large and a small peak cannot be claimed as a successful restoration of an object known to consist of two equal components [14]-[15]. Let this constraint be $\ell_{a} \leq \ell \leq\left(1-\ell_{a}\right)$, where $\ell_{a}$ is the smallest allowable value of $\ell$. Setting $\ell_{a}$ equal to 0.5 forces the two estimated peak intensities to be exactly the same. However, this constraint seems to be too strict, since when an estimate showing two components with slightly different amplitudes is found, the two components may be considered resolved. The choice of $\ell_{a}$ will be further discussed in Section IV.

Next suppose that the one-component model

$$
a f^{2}\left(x_{n}-b\right)
$$

is fitted with respect to $(a, b)$ to the same observations and that $(\hat{a}, \hat{b})$ is the so-called one-component solution, that is, the minimum of the corresponding LS criterion

$$
J_{1}(a, b)=\sum_{n} d_{n}^{2}(a, b)
$$

where $d_{n}(a, b)=w_{n}-a f^{2}\left(x_{n}-b\right)$. Then it can be shown that in the parameterization of $J_{2}\left(a, \ell, b_{1}, b_{2}\right)$ the point $(\hat{a}, \hat{b})$ is represented by the points

$$
(\bar{a}, \ell, \hat{b}, \hat{b})
$$

for all $\hat{a}$ and $\bar{a}$ satisfying

$$
\bar{a}=\hat{a} /\left(\ell^{2}+(1-\ell)^{2}+2 \gamma \ell(1-\ell)\right)
$$

for any $\ell$. Notice that the location parameters in (5) are equal. It can be shown that the points (5) are stationary points of $J_{2}\left(a, \ell, b_{1}, b_{2}\right)$. Stationary points of a function are defined as points where the gradient is equal to zero. From now on, the points (5) will be called one-component stationary points. It can be shown that they are either saddle points or minima. Which of both types occurs depends on the particular set of observations used. The occurrence of both types will be discussed in detail in the next section.

\section{Classification OF THE OBSERVATIONS}

First, consider the case that (5) is a saddle point. This implies that (5) is a maximum in at least one coordinate. Hence, there are points different from (5) where $J_{2}\left(a, \ell, b_{1}, b_{2}\right)$ has a smaller value. It can be shown that, as opposed to at point (5), at these points, $\hat{b}_{1}$ is not equal to $\hat{b}_{2}$. Consequently, the absolute minimum of $J_{2}\left(a, \ell, b_{1}, b_{2}\right)$ will represent two distinct components. Close to this absolute minimum and the saddle point $(5), J_{2}\left(a, \ell, b_{1}, b_{2}\right)$ has always a further relative minimum. In what follows this local structure of the least-squares criterion will be called the two-minima onesaddle-point (TMOS) structure. Outside the neighborhood of this cluster of three stationary points, other stationary points may exist, but they are irrelevant to the purposes of this paper because, as a rule, they represent a bad fit and/or parameter values that are physically meaningless. Depending on the particular set of observations used, the point (5) can also be a (possibly relative) minimum. Accordingly, the Euclidean $N$-space of the observations can be divided into two regions, separated from each other by a hypersurface. For sets of observations in the one region the point (5) is a saddle point, whereas for sets of observations in the other region the point (5) is a (possibly relative) minimum. It may be shown that the hypersurface mentioned above is identical with or very close to another hypersurface called the bifurcation set [16]. The bifurcation set separates the observations for which (5) is locally the only minimum from all other observations. At the bifuraction set the TMOS structure discontinuously changes into a one-minimum (OM) structure. Such discontinuous structural changes are called singularities and they are the subject of catastrophe and singularity theory [17]. The two point sources can not be resolved from sets of observations located on the side of the bifurcation set associated with the OM structure, since then the solutions for the locations $\hat{b}_{1}$ and $\hat{b}_{2}$ exactly coincide. This implies that fitting a two-component model results in a one-component solution. Consequently, the bifurcation set can be regarded as the resolution limit attainable by model fitting. The region between the bifurcation set and the hypersurface mentioned earlier corresponds to a two-minima one-saddle point structure with one minimum being the point (5). However, it follows from singularity theory that, for any PSF, the minima are so close that the point (5) may be safely taken as the absolute minimum [16], [17]. Consequently, between the hypersurfaces the components may be considered unresolved. The conclusion is that the hypersurface separating observations for which the point (5) is a saddle point from those for which it is a minimum also separates the observations from which the components can be resolved from those from which they cannot. Therefore, this hypersurface is taken as the resolution limit and will be addressed as such from now on.

The question how to compute this resolution limit will be discussed in the next section. In this section it will also be shown how to find out on which side of the resolution limit a particular set of observations is located or, equivalently, if the components can be resolved from these observations.

\section{Computation OF THE RESOlution Limit}

It can easily be shown that at the points (5), the value of $J_{2}\left(a, \ell, b_{1}, b_{2}\right)$ is for all $\ell$ equal to $J_{1}(\hat{a}, \hat{b})$. Hence, if there is any $\ell$ for which the model fitting solution $\left(\hat{a}, \ell, \hat{b}_{1}, \hat{b}_{2}\right)$ is different from (5), i.e., for which (5) is a saddle point, the value of $J_{2}\left(a, \ell, b_{1}, b_{2}\right)$ at $\left(\hat{a}, \ell, \hat{b}_{1}, \hat{b}_{2}\right)$ must be smaller than $J_{1}(\hat{a}, \hat{b})$. Since the best fit is taken as the solution, this means that resolution is always possible unless the points (5) are minima for all relevant $\ell$. The nature of the stationary points (5) depends on the signs of the eigenvalues of the matrix of second order derivatives, the Hessian matrix, of the criterion $J_{2}\left(a, \ell, b_{1}, b_{2}\right)$ with respect to the parameters $\left(a, b_{1}, b_{2}\right)$, evaluated at (5). In order to get more insight in the eigenstructure of this matrix, the coordinates are subsequently linearly transformed into

$$
\left(a r, \frac{\ell(\ell+\gamma(1-\ell)) b_{1}+(1-\ell)((1-\ell)+\gamma \ell) b_{2}}{r}, b_{2}-b_{1}\right)
$$

with $r=\left(\ell^{2}+(1-\ell)^{2}+2 \gamma \ell(1-\ell)\right)$. In these coordinates, the Hessian matrix $\mathrm{H}_{2}$ is a $3 \times 3$ block diagonal matrix, which 
is described by

$$
H_{2}=\operatorname{diag}\left(H_{1} \eta_{\ell}\right)
$$

where $H_{1}$ is the Hessian matrix of $J_{1}(a, b)$, evaluated at the minimum $(\hat{a}, \hat{b})$. Therefore, this matrix is positive definite. Consequently, the nature of the one-component stationary points (5) is fully determined by the sign of the scalar $\eta_{\ell}$, which is described by

$$
\eta_{\ell}=\left(4 \hat{a} \ell(1-\ell) / r^{2}\right) \rho_{\ell}
$$

where

$$
\rho_{\ell}=\ell(1-\ell)\left(\gamma^{2}-1\right) \chi-((1-\ell)+\gamma \ell)(\ell+\gamma(1-\ell)) \psi
$$

in which

$$
\chi=\sum_{n} d_{n}\left(\frac{\partial f_{n}}{\partial b}\right)^{2} \text { and } \psi=\sum_{n} d_{n} \frac{\partial^{2} f_{n}}{\partial b^{2}} f_{n}
$$

with $f_{n}=f\left(x_{n}-b\right)$ and $d_{n}=w_{n}-a f_{n}^{2}$. The quantities $d_{n}$, $f_{n}$, and the derivatives of $f_{n}$ are evaluated at $(\hat{a}, \hat{b})$. The first factor in (9) is always positive. Therefore, an analysis of the sign of $\eta_{\ell}$ can be replaced by an analysis of the sign of $\rho_{\ell}$. If $\rho_{\ell}$ is negative, the point (5) is a saddle point. If $\rho_{\ell}$ is positive, the point (5) is a minimum. To find out if the components can be resolved from a given set of observations, firstly the onecomponent model (3) is fitted. Secondly, the one-component solution $(\hat{a}, \hat{b})$ is substituted in (10) and the sign of the resulting value of $\rho_{\ell}$ is assessed for all relevant values of $\ell$.

For each $\ell$, a resolution limit can be defined. This resolution limit is constituted by the two equations obtained by putting the gradient of $J_{1}(a, b)$ with respect to $(a, b)$ equal to zero and the equation $\rho_{\ell}=0$. This results in three equations in the variables $(\hat{a}, \hat{b})$ and $\left(w_{1}, \ldots, w_{N}\right)$. After hypothetical elimination of $(\hat{a}, \hat{b})$, one equation in the variables $\left(w_{1}, \ldots, w_{N}\right)$ results, defining a hypersurface in the Euclidean $N$-space of the observations. Notice that this resolution limit is differently located for different values of $\ell$. Since the condition $\rho_{\ell}=0$ is linear in the observations and, for realistic values of the SNR, the one-component solution $(\hat{a}, \hat{b})$ will hardly vary under influence of the noise, the resolution limits are approximately hyperplanes. Simulation experiments have affirmed the high accuracy of this approximation. Now, for a given set of observations, resolution is impossible if for all $\ell$ this set is located on the side of the resolution limit associated with the OM structure. That is, resolution is impossible if $\rho_{\ell}$ is positive for all $\ell$. It can easily be shown that this is the case if both $\rho_{0.5}$ and $\rho_{\ell_{a}}$ are positive, since it follows from (10) that $\rho_{\ell}$ as a function of $\ell$ is a parabola with its extreme value at $\ell=0.5$. Consequently, we can define the overall resolution limit as the set of all sets of observations satisfying

$$
\left\{\rho_{\ell_{a}}=0 \wedge \rho_{0.5} \geq 0\right\} \vee\left\{\rho_{0.5}=0 \wedge \rho_{\ell_{a}} \geq 0\right\} .
$$

For a Gaussian PSF the analysis can be drastically simplified, since in this case $\chi=\psi$, taking into account that $(\hat{a}, \hat{b})$ is a stationary point of $J_{1}(a, b)$. Equation (10) can now be described as $\rho_{\ell}=F_{\ell}$, with

$$
F_{\ell}=\ell(1-\ell)\left(\gamma^{2}-1\right)-((1-\ell)+\gamma \ell)(\ell+\gamma(1-\ell)) \text {. }
$$

It follows from (13) that $F_{\ell}$ is a concave parabola having its minimum at $\ell=0.5$, at which $F_{1 / 2}=-1 / 2(\gamma+1) \leq 0$, for all $\gamma$. The roots of $F_{\ell}$ are given by

$$
\ell_{1,2}=1 / 2 \pm 1 / 2 \sqrt{(1+\gamma) /(1-\gamma)}
$$

Resolution is impossible if $\rho_{\ell}$ is positive for all relevant values of $\ell$. This is the case if i) $F_{\ell}$ has no roots on the interval $\left(\ell_{a},\left(1-\ell_{a}\right)\right)$ and ii) $\chi<0$.

Next, consider the value of $\ell_{a}$. Adopting the peak-to-peak intensity ratio constraint $2 / 3 \leq \ell^{2} /(1-\ell)^{2} \leq 3 / 2$ [14], [15] yields $\ell_{a} \approx 0.45$ and $0.45 \leq \ell \leq 0.55$. Then it follows from (14) that $F_{\ell}$ has no roots on the interval $(0.45,0.55)$ if $\gamma>-0.98$. Consequently, for a Gaussian PSF resolution would be impossible if i) $\gamma>-0.98$ and ii) $\chi<0$. Notice that condition $i)$ is rarely if ever violated. Putting the constraint $1 / 2 \leq \ell^{2} /(1-\ell)^{2} \leq 2$ would yield $\ell_{a} \approx 0.41$ and condition (i) would be replaced by $\gamma>-0.94$.

\section{Probability of Resolution in the Presence of Noise}

The purpose of this section is to show that if a priori knowledge about the statistical properties of the observations is available, this can be used to derive the probability of resolution as a function of the SNR. Suppose that $N$ independent observations $w_{1}, \ldots, w_{N}$ are available and that these observations can be described by their expectations $E\left[w_{n}\right]$ and variances $\operatorname{Var}\left\{w_{n}\right\}=\sigma_{n}^{2}$, respectively. $E[\cdot]$ and $\operatorname{Var}\{\cdot\}$ denote the expectation and variance operators, respectively. The standard definition of SNR, expressed in decibels, is given by

$$
\mathrm{SNR}=10^{10} \log \left(\sum_{n} E\left[w_{n}\right]^{2} / \sum_{n} \sigma_{n}^{2}\right) .
$$

In order to compute the probability of resolution when fitting the model (1) to the observations, we have to consider the statistical properties of the scalar (10), which depends on the one-component solution $(\hat{a}, \hat{b})$ and $\ell$. It is observed that $J_{1}(a, b)$ is quadratic in $a$. Therefore, the first-order partial derivative of $J_{1}(a, b)$ with respect to a is linear in this parameter. If this derivative is put equal to zero, a linear equation in the LS solution for a is obtained [18]. Solving this equation yields

$$
\hat{a}=\sum_{n} w_{n} f_{n}^{2}(\hat{b}) / \sum_{n} f_{n}^{4}(\hat{b}) .
$$

Unlike $a$, the location parameter $b$ occurs nonlinearly in the one-component model (3). Therefore, there exists no closed form solution for $b$. It must be determined iteratively by numerical nonlinear minimization of $J_{1}(a, b)$. However, the SNR is supposed to be sufficiently large to assume that the solution $\hat{b}$ will hardly vary under the influence of the noise. Furthermore, due to the functional shape of most conventional PSF's, the terms $\chi$ and $\psi$ in (10) will hardly vary with slight changes in $\hat{b}$. Therefore, $\hat{b}$ may be taken as a constant in the following analysis. Its value is set equal to the LS estimate obtained from fitting the model (3) to the $E\left[w_{n}\right]$. Simulation results have shown that this simplification is justified. Then it 
follows from (16) that the expectation and the variance of $\hat{a}$ are given by

$$
E[\hat{a}]=\frac{\sum_{n} E\left[w_{n}\right] f_{n}^{2}(\hat{b})}{\sum_{n} f_{n}^{4}(\hat{b})}, \quad \operatorname{Var}\{\hat{a}\}=\frac{\sum_{n} f_{n}^{4}(\hat{b}) \sigma_{n}^{2}}{\left(\sum_{n} f_{n}^{4}(\hat{b})\right)^{2}}
$$

respectively. As was shown in Section IV, resolution is impossible if both $\rho_{0,5}$ and $\rho_{\ell_{a}}$ are positive. If the number of observations is sufficiently large for the central limit theorem to hold [19], it follows from (10) and (11) and the considerations above that $\rho_{0.5}$ and $\rho_{\ell_{a}}$ are both normally distributed. Then, $\left(\rho_{0.5}, \rho_{\ell_{a}}\right)$ has a bivariate normal distribution $f$ given by [19]

$$
f\left(\rho_{0 . \tilde{z}}, \rho_{\ell_{a}}\right)=\frac{1}{2 \pi|\Phi|^{1 / 2}} \exp \left(-1 / 2(\rho-\mu)^{T} \Phi^{-1}(\rho-\mu)\right)
$$

where $\rho^{T}=\left(\rho_{0.5}, \rho_{\ell_{a}}\right), \mu^{T}=\left(E\left[\rho_{0.5}\right], E\left[\rho_{\ell_{a}}\right]\right)$ and $\Phi$ is the $2 \times 2$ covariance matrix of $\left(\rho_{0.5}, \rho_{\ell_{a}}\right)$. The computation of $\mu$ and $\Phi$ is straightforward and is presented in the Appendix. The probability of resolution is now given by

$$
1-\int_{0}^{\infty} \int_{0}^{\infty} f\left(\rho_{0.5}, \rho_{\ell_{a}}\right) d \rho_{0.5} d \rho_{\ell_{a}} .
$$

It was shown in Section IV that for a Gaussian PSF, resolution is impossible if i) $F_{\ell}$ has no roots on the interval $\left(\ell_{a},\left(1-\ell_{a}\right)\right)$ and ii) $\chi<0$. If the number of observations is sufficiently large for the central limit theorem to hold, it follows from (11) that $\chi \sim N(E[\chi], \operatorname{Var}\{\chi\})$, where $E[\chi]$ and $\operatorname{Var}\{\chi\}$ are derived in the Appendix. Then, provided that condition i) is fulfilled, the probability of resolution is given by

$$
\frac{\int_{0}^{\infty} \exp \left(-\frac{(\chi-E[\chi]]^{2}}{2 \operatorname{Var}\{\chi\}}\right) d \chi}{\sqrt{2 \pi \operatorname{Var}\{\chi\}}}=\frac{1}{2}\left(1+\operatorname{Erf}\left(\frac{E[\chi]}{\sqrt{2 \operatorname{Var}\{\chi\}}}\right)\right)
$$

where $\operatorname{Erf}(\cdot)$ represents the error function.

\section{NUMERICAL EXAMPLE}

This experiment tests the expressions (19) and (20) when used to predict the probability of resolution. For this purpose observations on two overlapping coherent components were generated, described by

$$
w_{n}=g_{n}\left(\alpha, \lambda, \beta_{1}, \beta_{2}\right)+\varepsilon_{n}
$$

where the model $g$ is given by (1). Furthermore, $\alpha=4, \lambda=$ $0.5, \beta_{1}=-0.05, \beta_{2}=0.05, \gamma=0.4, x_{n}=-0.01+(n-$ 11) $\times 0.4$, with $n=1, \ldots, 21$ and $\varepsilon_{n} \sim N\left(0, \sigma_{\varepsilon}^{2}\right)$. In a number of simulations $\sigma_{\varepsilon}$ is stepwise increased from 0.002 to 0.02 . For each value of $\sigma_{\varepsilon} 10000$ sets of observations are generated and each time the model (1) is fitted to the observations with respect to $\left(a, b_{1}, b_{2}\right)$ for various values of $\ell$ on the interval $(0.45,0.55)$ and the best fit is taken as a solution. This has been done for a sinc and a Gaussian PSF described by $f(x)=\sin (x) / x$ and $f(x)=\exp \left\{-1 / 4 x^{2}\right\}$, respectively. For each $\sigma_{\varepsilon}$ the percentage of the simulations providing resolution of the components, as well as the percentages predicted by (19) and (20) (for a sinc and a Gaussian PSF, respectively) are determined. Fig. 1 shows both the predicted and actually

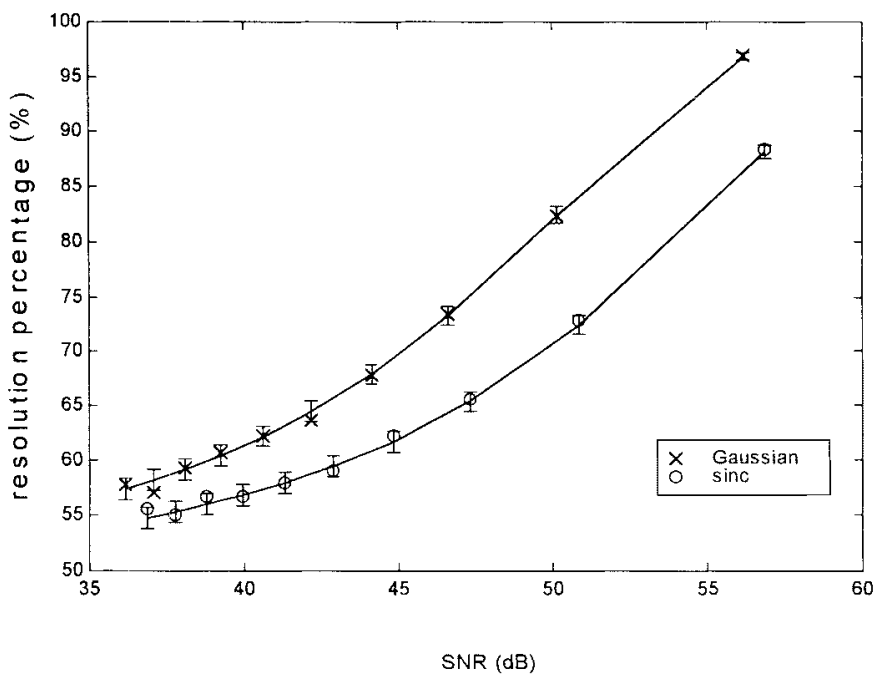

Fig. 1. Results of the numerical example of Section VI: predicted (curves) and actually obtained (" $x$ " and " $o$ ") percentages of resolution for a Gaussian and sinc PSF. The errorbars represent the $95 \%$ confidence regions.

obtained percentages of resolution as a function of the SNR, including the $95 \%$ confidence regions of the predicted values. It turns out that (19) and (20) provide accurate predictions.

\section{CONCLUSION}

Accurate knowledge of the resolution limits fundamentally imposed by systematic and nonsystematic errors is of obvious relevance for the design of imaging systems. In this paper, a model fitting based definition of resolution has been presented and discussed. It has been shown that two distinct types of sets of observations can be distinguished. For the one type, the model fitting solutions for the locations are distinct and the components are resolved. For the other type, the solutions exactly coincide and the components are not resolved. Which type occurs depends on the particular set of observations used. It has also been explained how these types can be determined for any two-component model. Furthermore, for statistical observations, the probability of resolution can be computed as a function of the SNR. This probability can be used to compare the performance of different imaging systems.

Finally, it should be noticed that the theory presented in this paper is not confined to strictly 1-D imaging systems. For 2-D imaging similar results have been found [20]-[22].

\section{APPENDIX}

First, a shorthand notation is introduced:

$$
f_{n}=f\left(x_{n}-\hat{b}\right) \text { and } f_{n}^{(k)}=\frac{\partial^{k} f_{n}(\hat{b})}{\partial b^{k}}, \quad k \in\{1,2\} .
$$

Furthermore, the following constants are defined

$$
\begin{aligned}
& c_{11}=\frac{\left(1-\gamma^{2}\right)}{4}, \quad c_{21}=\frac{(1+\gamma)^{2}}{4}, \\
& c_{12}=\left(1-\gamma^{2}\right) \ell_{a}\left(1-\ell_{a}\right), \\
& c_{22}=\left(\left(1-\ell_{a}\right)+\gamma \ell_{a}\right)\left(\ell_{a}+\gamma\left(1-\ell_{a}\right)\right) .
\end{aligned}
$$


It follows from (10), (11), and (17) that the expected values of $\rho_{0.5}$ and $\rho_{\ell_{a}}$ are given by

$$
\begin{aligned}
E\left[\rho_{i}\right]= & -c_{1 i} \sum_{n}\left(E\left[w_{n}\right]-E[\hat{a}] f_{n}^{2}\right)\left(f_{n}^{(1)}\right)^{2} \\
& -c_{2 i} \sum_{n}\left(E\left[w_{n}\right]-E[\hat{a}] f_{n}^{2}\right) f_{n} f_{n}^{(2)}
\end{aligned}
$$

with $i \in\{1,2\}, \rho_{1}=\rho_{0,5}$ and $\rho_{2}=\rho_{\ell_{a}}$. The elements of the $2 \times 2$ covariance matrix $\Phi$ of $\left(\rho_{0 . \tilde{5}}, \rho_{\ell_{a}}\right)$ in (18) are given by

$$
\begin{aligned}
\Phi_{i j}= & \operatorname{Cov}\left(\rho_{i}, \rho_{j}\right)=c_{1 i} c_{1 j}\left(\sum_{n} \sigma_{n}^{2}\left(f_{n}^{(1)}\right)^{4}\right. \\
& +\operatorname{Var}\{\hat{a}\}\left(\sum_{n} f_{n}^{2}\left(f_{n}^{(1)}\right)^{2}\right)^{2} \\
& \left.-2 \sum_{n} \operatorname{Cov}\left(\hat{a}, w_{n}\right)\left(f_{n}^{(1)}\right)^{2} \sum_{n} f_{n}^{2}\left(f_{n}^{(1)}\right)^{2}\right) \\
& +c_{2 i} c_{2 j}\left(\sum_{n} \sigma_{n}^{2} f_{n}^{2}\left(f_{n}^{(2)}\right)^{2}+\operatorname{Var}\{\hat{a}\}\left(\sum_{n} f_{n}^{3} f_{n}^{(2)}\right)^{2}\right. \\
& \left.-2 \sum_{n} \operatorname{Cov}\left(\hat{a}, w_{n}\right) f_{n} f_{n}^{(2)} \sum_{n} f_{n}^{3} f_{n}^{(2)}\right) \\
& +\left(c_{1 i} c_{2 j}+c_{1 j} c_{2 i}\right)\left(\sum_{n} f_{n}\left(f_{n}^{(1)}\right)^{2} f_{n}^{(2)} \sigma_{n}^{2}\right. \\
& -\sum_{n} \operatorname{Cov}\left(\hat{a}, w_{n}\right)\left(f_{n}^{(1)}\right)^{2} \sum_{n} f_{n}^{3} f_{n}^{(2)} \\
& -\sum_{n} \operatorname{Cov}\left(\hat{a}, w_{n}\right) f_{n} f_{n}^{(2)} \sum_{n} f_{n}^{2}\left(f_{n}^{(1)}\right)^{2} \\
& \left.+\operatorname{Var}\{\hat{a}\} \sum_{n} f_{n}^{2}\left(f_{n}^{(1)}\right)^{2} \sum_{n} f_{n}^{3} f_{n}^{(2)}\right)
\end{aligned}
$$

where $i, j \in\{1,2\}, \rho_{1}=\rho_{0.5}$ and $\rho_{2}=\rho_{\ell_{a}}$. Furthermore,

$$
\operatorname{Cov}\left(\hat{a}, w_{n}\right)=f_{n}^{2} \sigma_{n}^{2} / \sum_{n} f_{n}^{4}
$$

The mean and the variance of $\chi$ in (20) are given by

$$
E[\chi]=\sum_{n}\left(E\left[w_{n}\right]-E[\hat{a}] f_{n}^{2}\right)\left(f_{n}^{(1)}\right)^{2}
$$

and

$$
\begin{aligned}
\operatorname{Var}\{\chi\}= & \sum_{n} \sigma_{n}^{2}\left(f_{n}^{(1)}\right)^{4}+\operatorname{Var}\{\hat{a}\}\left(\sum_{n} f_{n}^{2}\left(f_{n}^{(1)}\right)^{2}\right)^{2} \\
& -2 \sum_{n} \operatorname{Cov}\left(\hat{a}, w_{n}\right)\left(f_{n}^{(1)}\right)^{2} \sum_{n} f_{n}^{2}\left(f_{n}^{(1)}\right)^{2}
\end{aligned}
$$

respectively.

\section{REFERENCES}

[1] V. Ronchi, "Resolving power of calculated and detected images," $J$. Opt. Soc. Amer., vol. 51, pp. 458-460, 1961.

[2] L. Rayleigh, "On the manufacture and theory of diffraction-gratings," Philos. Mag., vol. 47, pp. 81-93, pp. 193-205, 1874.

[3] J. W. Goodman, Statistical Optics. New York: Wiley, 1985, pp. 326-328.

[4] T. Orhaug, "On the resolution of imaging systems," Optica Acta, vol. 16, pp. 75-84, 1969.

[5] C. W. Helstrom, "Resolvability of objects from the standpoint of statistical parameter estimation," J. Opt. Soc. Amer., vol. 60, pp. 659-666, 1970.

[6] W. T. Cathey, B. R. Frieden, W. T. Rhodes, and C. K. Rushforth, "Image gathering and processing for enhanced resolution," J. Opt. Soc. Amer., vol. 1 , pp. 241-250, 1984

[7] J. T. Reagan and T. J. Abatzoglou, "Model-based super-resolution CSO processing," in Proc. SPIE_Int. Soc. Optical Engineering, 1993, vol. 1954, pp. 204-218.

[8] A. van den Bos, "Optical resolution: An analysis based on catastrophe theory," J. Opt. Soc. Amer., vol. 4, pp. 1402-1406, 1987.

[9] A. J. den Dekker, "On two-point resolution in partially coherent light: A parameter estimation approach," in Proc. EUSIPCO-94, 7th European Signal Processing Conf., Edinburgh, Scotland, U.K., 1994, vol. III, pp. $1421-1424$

[10] L. J. van Vliet, "Grey-scale measurements in multi-dimensional digitized images," Ph.D. dissertation, Delft Univ. Press, Delft, The Netherlands, 1993.

[11] M. Born and E. Wolf, Principles of Optics. Oxford, U.K.: Pergamon, 1980.

[12] A. van den Bos and J. H. Swarte, "Resolvability of the parameters of multi-exponentials and other sum models," IEEE Trans. Signal Processing, vol. 41, pp. 313-322, 1993.

[13] J. H. Swarte, "Precision and resolution in spectroscopic model fitting," Ph.D. dissertation, Delft University Press, Delft, The Netherlands, 1992.

[14] L. B. Lucy, "Statistical limits to superresolution," Astron. Astrophys. vol. 261, pp. 706-710, 1992.

[15] $\ldots$,Resolution limits for deconvolved Images," Astron. J., vol. 104, no. 3, pp. 1260-1265, 1992.

[16] A. van den Bos, "Degeneracy in nonlinear least squares," in IEE Proc. D., 1981, vol. 128, no. 3, pp. 109-116.

[17] V. I. Arnol'd, Catastrophe Theory. Berlin, Germany: Springer, 1992.

[18] W. H. Lawton and E. A. Sylvestre, "Elimination of linear parameters in nonlinear regression," Technometrics, vol. 13, no. 3, pp. 461-467, 1971.

[19] A. Stuart and K. Ord, Kendall's Advanced Theory of Statistics, 6th ed. London, U.K.: E. Arnold, 1994.

[20] A. van den Bos, "Ultimate resolution: A mathematical framework," Ultramicroscopy, vol. 47, pp. 298-306, 1992.

[21] A. van den Bos and A. J. den Dekker, "Ultimate resolution in the presence of coherence," Ultramicroscopy, vol. 60, pp. 345-348, 1995.

[22] A. J. den Dekker, "Model-based optical two-point resolution," in Proc. ICASSP'96, 1996 Int. Conf. Acoustics, Speech, Signal Processing, Atlanta, GA, vol. IV, pp. 2395-2398.

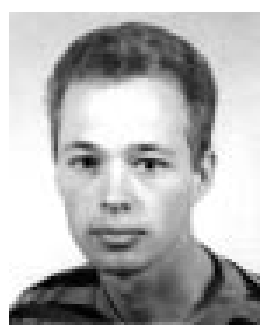

Arnold J. den Dekker was born in Vlaardingen, The Netherlands, in April 1969. He received the M.Sc. degree in applied physics from the Delft University of Technology, Delft, The Netherlands, in 1992 . He is presently pursuing the Ph.D. degree in applied physics at Delft University of Technology.

His main research interests are in applying parameter estimation to measurement problems in various fields of physics. 